\title{
Iterative Solution of the Normal-Equations Form of the Electric-Field Integral Equation ${ }^{\dagger}$
}

\author{
Özgür Ergül ${ }^{1}$ and Levent Gürel ${ }^{* 1,2}$ \\ ${ }^{1}$ Department of Electrical and Electronics Engineering \\ ${ }^{2}$ Computational Electromagnetics Research Center (BiLCEM) \\ Bilkent University, TR-06800, Bilkent, Ankara, Turkey \\ E-mail: \{ergul,lgurel\}@ee.bilkent.edu.tr
}

\section{Introduction}

Among different surface formulations of electromagnetic scattering problems, electric-field integral equation (EFIE) is successfully used in the formulations of the problems involving conducting geometries modelled by open surfaces [1]. On the other hand, EFIE usually produces ill-conditioned matrix equations that are difficult to solve iteratively [2]. Especially, as the problem size gets larger and the dimensions of the matrix equation grow, solutions with EFIE become extremely difficult, even when iterative techniques are used with acceleration methods, such as the multilevel fast multipole algorithm (MLFMA) [3] for the matrixvector multiplications. For efficient solutions, however, it is extremely important to reduce the number of iterations. In this paper, we show that transforming the original equations into normal equations improves the convergence of EFIE significantly. We present the solutions of EFIE by employing the least-squares QR (LSQR) algorithm [4], which corresponds to a stable application of the conjugate gradient (CG) algorithm on the normal equations. Despite the squaring of the condition number due to such a transformation into the normal equations, LSQR improves the convergence rate of the iterative solutions of EFIE and performs better than many other iterative algorithms that are commonly used in the literature. In addition to LSQR, we present the accelerated convergence of the normal equations in the context of the generalized minimal residual (GMRES) algorithm, where the memory requirement is reduced significantly due to the the improved convergence characteristics.

\section{Solutions of Normal Equations with MLFMA}

For conducting surfaces, EFIE can be written directly from the boundary condition for the tangential electric field as [1]

$$
\hat{\boldsymbol{t}} \cdot \int_{S} d \boldsymbol{r}^{\prime} \boldsymbol{J}\left(\boldsymbol{r}^{\prime}\right) \cdot\left(\overline{\mathbf{I}}-\frac{\nabla \nabla^{\prime}}{k^{2}}\right) g\left(\boldsymbol{r}, \boldsymbol{r}^{\prime}\right)=\frac{i}{k \eta} \hat{\boldsymbol{t}} \cdot \boldsymbol{E}^{\boldsymbol{i}}(\boldsymbol{r})
$$

in phasor notation with the $e^{-i w t}$ convention. In (1), $\hat{\boldsymbol{t}}$ is the tangential unit vector on the surface at the observation point $\boldsymbol{r}, \boldsymbol{E}^{\boldsymbol{i}}$ is the incident electric field, $\boldsymbol{J}$ is the electric current induced on the surface $S, k$ is the wavenumber, $\eta$ is the characteristic impedance of free space, and

$$
g\left(\boldsymbol{r}, \boldsymbol{r}^{\prime}\right)=\frac{e^{i k R}}{4 \pi R} \quad\left(R=\left|\boldsymbol{r}-\boldsymbol{r}^{\prime}\right|\right)
$$

denotes the free-space Green's function. By the simultaneous discretization of the geometry and EFIE, $N \times N$ matrix equation is obtained as

$$
\bar{Z} \cdot a=v,
$$

where $\boldsymbol{a}$ represents the unknown coefficient vector, $\overline{\boldsymbol{Z}}$ is the impedance matrix, and $\boldsymbol{v}$ is the excitation vector. For divergence-conforming functions, such as the Rao-Wilton-

\footnotetext{
${ }^{\dagger}$ This work was supported by the Scientific and Technical Research Council of Turkey (TUBITAK) under Research Grant 105E172, by the Turkish Academy of Sciences in the framework of the Young Scientist Award Program (LG/TUBA-GEBIP/2002-1-12), and by contracts from ASELSAN and SSM.
} 
Glisson (RWG) [5] and linear-linear (LL) [6] functions, matrix elements are derived as

$$
\begin{aligned}
Z_{m n} & =\int_{S_{m}} d \boldsymbol{r} \boldsymbol{t}_{m}(\boldsymbol{r}) \cdot \int_{S_{n}} d \boldsymbol{r}^{\prime} g\left(\boldsymbol{r}, \boldsymbol{r}^{\prime}\right) \boldsymbol{b}_{n}\left(\boldsymbol{r}^{\prime}\right) \\
& -\frac{1}{k^{2}} \int_{S_{m}} d \boldsymbol{r}\left[\nabla \cdot \boldsymbol{t}_{m}(\boldsymbol{r})\right] \int_{S_{n}} d \boldsymbol{r}^{\prime} g\left(\boldsymbol{r}, \boldsymbol{r}^{\prime}\right)\left[\nabla^{\prime} \cdot \boldsymbol{b}_{n}\left(\boldsymbol{r}^{\prime}\right)\right]
\end{aligned}
$$

for each $m, n=1,2, \ldots, N$. In (4), $\boldsymbol{b}_{n}$ and $\boldsymbol{t}_{m}$ represent the set of basis and testing functions with the spatial supports of $S_{n}$ and $S_{m}$, respectively.

For the iterative solutions of (3), matrix-vector multiplications are required at each iteration. These multiplications can be performed efficiently by the fast multipole method (FMM) as [7]

$$
Z_{m n}=\frac{i k}{(4 \pi)^{2}} \int d^{2} \hat{\boldsymbol{k}} \boldsymbol{F}_{C m}^{r e c}(\hat{\boldsymbol{k}}) T_{L}(k,|\boldsymbol{D}|, \hat{\boldsymbol{D}} \cdot \hat{\boldsymbol{k}}) \cdot \boldsymbol{F}_{C^{\prime} n}^{r a d}(\hat{\boldsymbol{k}}),
$$

where $\hat{\boldsymbol{k}}$ is the angular direction on the unit sphere, and

$$
T_{L}(k,|\boldsymbol{D}| \hat{\boldsymbol{D}} \cdot \hat{\boldsymbol{k}})=\sum_{l=0}^{L} i^{l}(2 l+1) h_{l}^{(1)}(k D) P_{l}(\hat{\boldsymbol{D}} \cdot \hat{\boldsymbol{k}})
$$

is the translation function written in terms of the spherical Hankel function of the first kind $h_{l}^{(1)}$ and the Legendre polynomial $P_{l}$. In (5), $\boldsymbol{F}_{C m}^{r e c}$ and $\boldsymbol{F}_{C^{\prime} n}^{r a d}$ represent the receiving and radiation patterns of the $m$ th testing and $n$th basis functions, respectively, with respect to reference points $C$ and $C^{\prime}$. The translation function in (6) evaluates the interaction between the basis and testing groups that are located at $C^{\prime}$ and $C$, respectively, and separated by

$$
\boldsymbol{D}=|\boldsymbol{D}| \hat{\boldsymbol{D}}=\boldsymbol{r}_{C}-\boldsymbol{r}_{C^{\prime}}
$$

Using a Galerkin scheme, the radiation and receiving patterns are calculated as

$$
\boldsymbol{F}_{C^{\prime} n}^{r a d}(\hat{\boldsymbol{k}})=\int_{S_{n}} d \boldsymbol{r}^{\prime} \exp \left[-i \boldsymbol{k} \cdot\left(\boldsymbol{r}^{\prime}-\boldsymbol{r}_{C^{\prime}}\right)\right](\boldsymbol{I}-\hat{\boldsymbol{k}} \hat{\boldsymbol{k}}) \cdot \boldsymbol{b}_{n}\left(\boldsymbol{r}^{\prime}\right)=\left[\boldsymbol{F}_{C m}^{r e c}(\hat{\boldsymbol{k}})\right]^{*},
$$

where the superscript ' $*$ ' denotes complex conjugation. For both RWG and LL functions, the integrals in (8) are evaluated analytically. To perform the matrix-vector multiplications by MLFMA, the scatterer is bounded by a computational cubic box, which is divided recursively into smaller boxes. This way, a tree-structure is formed, on which the calculations in (5) are performed in a multilevel scheme. Using MLFMA, the complexity of the matrixvector multiplications related to an $N \times N$ dense matrix equation is reduced from $O\left(N^{2}\right)$ to $O(N \log N)[3]$.

To obtain improved convergence in the iterative solutions of EFIE, we construct the normal equations as

$$
\overline{\boldsymbol{Z}}^{H} \cdot \overline{\boldsymbol{Z}} \cdot \boldsymbol{a}=\left\{\overline{\boldsymbol{Z}}^{T}\right\}^{*} \cdot \boldsymbol{a}=\overline{\boldsymbol{Z}}^{H} \cdot \boldsymbol{v},
$$

where ' $T$ ' represents the transpose operation. We note that the matrix of the normal equation, i.e., $\overline{\boldsymbol{Z}}^{H} \cdot \overline{\boldsymbol{Z}}$, is a Hermitian matrix. Using a Galerkin scheme, the EFIE matrix becomes symmetric so that only the complex conjugate operation is required in (9). Then, the original MLFMA can be employed to calculate the matrix-vector multiplications related to the complex-conjugate matrix as

$$
\boldsymbol{y}=\overline{\boldsymbol{Z}}^{*} \cdot \boldsymbol{x}=\left[\overline{\boldsymbol{Z}} \cdot \boldsymbol{x}^{*}\right]^{*},
$$

where only the input $(\boldsymbol{x})$ and output $(\boldsymbol{y})$ vectors are required to be modified. In the case of non-Galerkin EFIE, however, transpose operation is required in (9), which can be implemented carefully by reversing the steps of the ordinary MLFMA [8]. 
Table 1: Solutions of the Scattering Problems Involving a Patch Geometry of Various Sizes

\begin{tabular}{|c|c|c|c|c|c|c|c|}
\hline & & \multicolumn{3}{|c|}{ GMRES } & \multicolumn{3}{c|}{ GMRES-NE } \\
\hline Size & Unknowns & Iters & Time & Memory & Iters & Time & Memory \\
\hline \hline $12 \lambda \times 12 \lambda$ & 49,200 & 620 & $567 \mathrm{sec}$ & $60 \mathrm{MB}$ & 254 & $442 \mathrm{sec}$ & $43 \mathrm{MB}$ \\
\hline $18 \lambda \times 18 \lambda$ & 111,405 & 776 & $2566 \mathrm{sec}$ & $112 \mathrm{MB}$ & 335 & $2143 \mathrm{sec}$ & $67 \mathrm{MB}$ \\
\hline $20 \lambda \times 20 \lambda$ & 137,792 & 793 & $2795 \mathrm{sec}$ & $138 \mathrm{MB}$ & 360 & $2408 \mathrm{sec}$ & $79 \mathrm{MB}$ \\
\hline $25 \lambda \times 25 \lambda$ & 215,338 & 877 & $4658 \mathrm{sec}$ & $210 \mathrm{MB}$ & 413 & $4105 \mathrm{sec}$ & $117 \mathrm{MB}$ \\
\hline $30 \lambda \times 30 \lambda$ & 310,383 & 991 & $6518 \mathrm{sec}$ & $312 \mathrm{MB}$ & 467 & $5553 \mathrm{sec}$ & $169 \mathrm{MB}$ \\
\hline
\end{tabular}

\section{Results}

To show the improved convergence of EFIE by the construction of the normal equations, we present the results of two scattering problems involving a half sphere (HS) and an open prism (OP), as depicted in Fig. 1. The HS problem is solved at $2 \mathrm{GHz}$ and $7.5 \mathrm{GHz}$, while the $\mathrm{OP}$ problem is solved at $5 \mathrm{GHz}$ and $16 \mathrm{GHz}$. For the lower frequencies, discretizations of the problems with about $\lambda / 10$ triangulation lead to 9,911 and 11,351 unknowns for the HS and the OP problems, respectively ("small" problems). For the higher frequencies, similar discretizations with $\lambda / 10$ mesh size lead to 116,596 and 127,925 unknowns ("large" problems). We use RWG functions in a Galerkin scheme and employ a parallel MLFMA implementation to perform the matrix-vector multiplications. For each geometry, we solve two scattering problems involving the plane-wave excitations described in Fig. 1. As an iterative solver, we employ the LSQR algorithm, which performs the CG solutions of the transformed equation in (9) in an implicit and stable way. In addition to LSQR, we also use other Krylov subspace methods that are commonly used in the literature, i.e., GMRES, conjugate gradient squared (CGS), biconjugate gradient (BiCG), stabilized BiCG (BiCGSTAB), and transpose-free quasi-minimal residual (TFQMR) [9]. Fig. 2 presents the processing time required by various iterative algorithms for the solutions of the scattering problems. The processing times are measured on an 8-way SMP server with dual-core AMD Opteron processors for the convergence of the residual error under $10^{-6}$. Maximum and minimum numbers of the matrix-vector multiplications required by the iterative algorithms are also indicated in the plots. We observe that LSQR outperforms the other iterative algorithms, except for GMRES with a restart parameter of 1000. However, GMRES requires considerably larger memory than all other algorithms. For example, for the solution of the large HS problem, the memory requirement of GMRES is about 140 MB per processor, while it is only $1-1.5 \mathrm{MB}$ for other algorithms. Consequently, leaving the memory-hungry GMRES aside, LSQR requires the minimum processing time and it is the most efficient algorithm among those considered in Fig. 2

The improved convergence provided by LSQR is due to the favorable properties of the normal equations in (9) for EFIE. To further present the benefits of the normal equations, we solve scattering problems involving a square patch geometry with various sizes from $12 \lambda \times 12 \lambda$ to $30 \lambda \times 30 \lambda$. The patch is located in the $x-y$ plane and illuminated by a plane wave propagating in the $-z$ direction. Table 1 lists the number of unknowns, number of iterations for $10^{-6}$ residual error, solution time, and the memory requirement per processor when a no-restart GMRES algorithm is employed for the solutions. We observe that the convergence of the normal equations (denoted by GMRES-NE) are significantly faster than the convergence of the direct solutions of EFIE by GMRES. However, GMRES-NE requires two matrix-vector multiplications per iterations so that the acceleration of solutions is not as significant as the reduction in the number of iterations. On the other hand, GMRES-NE requires considerably lower memory than the direct solution of EFIE due to the reduced number of iterations. Reduced memory requirement is essential for the solution of large scattering problems. 

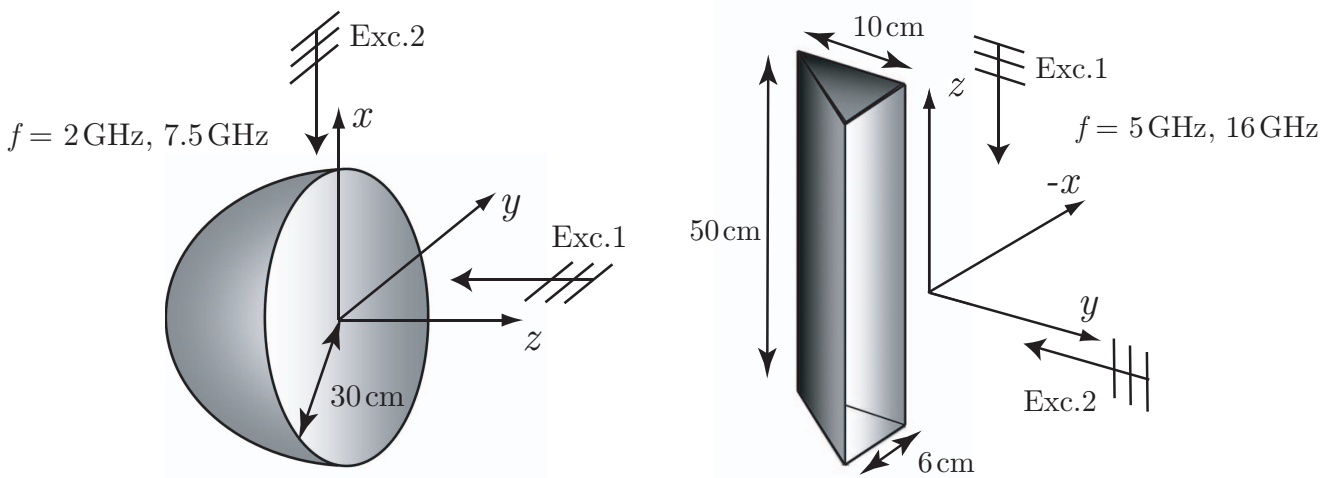

Figure 1: Scattering problems involving a half sphere and an open prism.
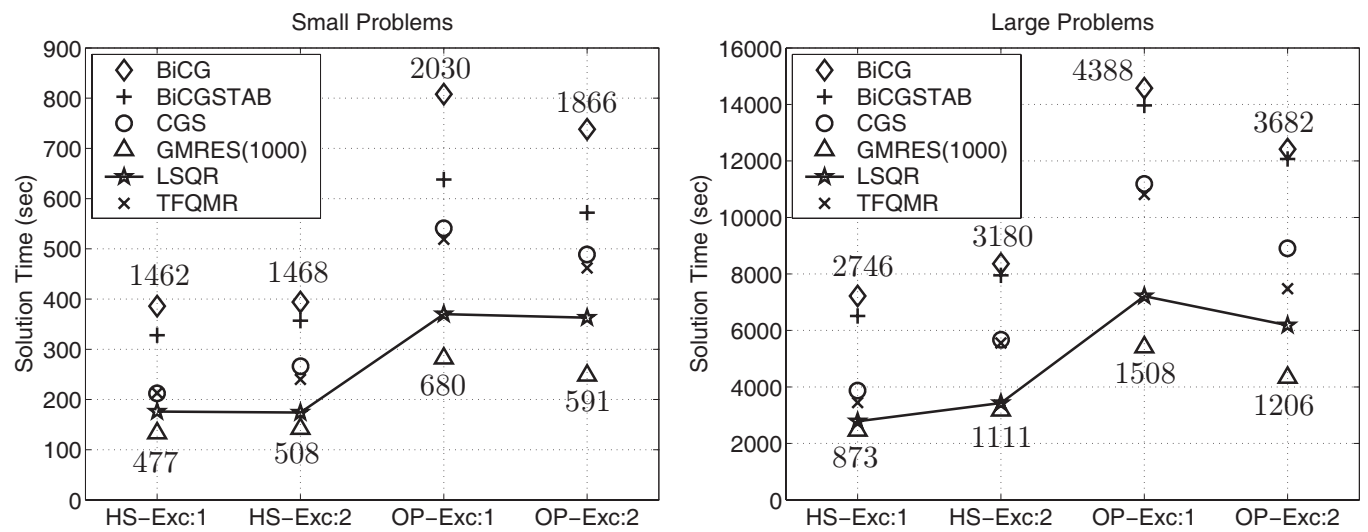

Figure 2: Processing times required by various iterative algorithms for the MLFMA solutions of the scattering problems described in Fig. 1. Maximum and minimum numbers of the matrix-vector multiplications are also indicated.

\section{References}

[1] A. J. Poggio and E. K. Miller, "Integral equation solutions of three-dimensional scattering problems," in Computer Techniques for Electromagnetics, R. Mittra, Ed. Oxford: Permagon Press, 1973, Chap. 4.

[2] L. Gürel and Ö. Ergül, "Comparisons of FMM implementations employing different formulations and iterative solvers," in Proc. IEEE Antennas and Propagation Soc. Int. Symp., vol. 1, 2003, pp. 19-22.

[3] C.-C. Lu and W. C. Chew, "Multilevel fast multipole algorithm for electromagnetic scattering by large complex objects," IEEE Trans. Antennas Propagat., vol. 45, no. 10, pp. 1488-1493, Oct. 1997.

[4] C. C. Paige and M. A. Saunders, ACM Trans. Math. Software, vol. 8, pp. 43-71, March 1982.

[5] S. M. Rao, D. R. Wilton, and A. W. Glisson, "Electromagnetic scattering by surfaces of arbitrary shape," IEEE Trans. Antennas Propagat., vol. AP-30, no. 3, pp. 409-418, May 1982.

[6] Ö. Ergül and L. Gürel, "Improving the accuracy of the magnetic field integral equation with the linear-linear basis functions," Radio Sci., vol. 41, RS4004, doi:10.1029/2005RS003307, 2006.

[7] W. C. Chew, J.-M. Jin, E. Michielssen, and J. Song, Fast and Efficient Algorithms in Computational Electromagnetics. Boston, MA: Artech House, 2001.

[8] Ö. Ergül and L. Gürel, "Iterative least-squares solution of the combined-field integral equation," 2005 IEEE AP-S International Symposium and USNC/URSI National Radio Science Meeting, Washington, DC, USA, July 2005.

[9] S. Balay, K. Buschelman, V. Eijkhout, W. D. Gropp, D. Kaushik, M. G. Knepley, L. C. McInnes, B. F. Smith, and H. Zhang, PETSc Users Manual, Argonne National Laboratory, 2004. 